\title{
Strategische Devianz: Potenziale und Risiken ungehorsamer Ästhetik in der Kommunikation sozialer Bewegungen
}

\author{
Melanie Malczok
}

Keywords: Strategische Kommunikation, Devianz, Legitimität, Hambacher Forst, Bildanalyse

\section{Abstract}

In der Frage danach, wohin sich eine Gesellschaft bewegen soll, kommen nicht alle Stimmen gleichermaßen zu Wort. Um dem zu begegnen, nutzen insbesondere ressourcenschwache Akteure im Kampf um öffentliche Sichtbarkeit kreative und kontroverse Mittel. Dabei gehen sie jedoch das Risiko ein, dass ihre Handlungen als normverletzend, also deviant wahrgenommen werden. Dies kann die öffentlich zugeschriebene Legitimität der jeweiligen Positionen bedrohen, die jedoch notwendig ist, um Deutungshoheit für bestimmte Themen zu erlangen und gesellschaftlichen Wandel zu erreichen. Der Beitrag differenziert Devianz in funktionale und dysfunktionale Formen der Normenabweichung. Erstere stellt eine progressive Herausforderung des Gegebenen dar, während letztere eher als systemzersetzend wahrgenommen wird. Das Beispiel der visuellen Kommunikation um die Rodungsarbeiten im Hambacher Forst verdeutlicht, welche Inszenierungsstrategien der Devianz zwar aufmerksamkeitsförderlich, jedoch potenziell legitimitätsbedrohend für Akteure sind und welche Formen die Zuschreibung von Legitimität tatsächlich befördern können.

Melanie Malczok: Strategische Devianz: Potenziale und Risiken ungehorsamer Ästhetik in der Kommunikation sozialer Bewegungen. In: Nils S. Borchers, Selma Güney, Uwe Krüger und Kerem Schamberger (Hrsg.): Transformation der Medien - Medien der Transformation. Verhandlungen des Netzwerks Kritische Kommunikationswissenschaft. Frankfurt am Main: Westend 2021. DOI: https://doi.org/10.53291/ZBAM1601.

Melanie Malczok I Hochschule Osnabrück I m.malczok@hs-osnabrueck.de 
Die Frage danach, wohin sich die Gesellschaft in Zukunft bewegen sollte, ist gesellschaftlich stark umkämpft. Kräfte, die auf Beharrung bestehender Institutionen pochen, stehen drängenden Forderungen nach Wandel gegenüber. Ort dieser Aushandlung ist die Öffentlichkeit. Sie hat die Funktion, der Gesellschaft einen Ort der Selbstthematisierung und Reflexion zur Verfügung zu stellen (Görke 2008; Wimmer 2007) und wird wesentlich von Journalismus hergestellt. Gleichzeitig spielen auch andere Akteure eine Rolle - im hier betrachteten Fall Organisationen, die als strategische beziehungsweise persuasive Kommunikatorinnen in Erscheinung treten und ihre Positionen diversen Öffentlichkeiten vermitteln wollen. Sie gestalten durch eigene Strategische Kommunikation (beispielsweise in den Sozialen Medien), aber auch in direktem Bezug auf den Journalismus Öffentlichkeit mit und versuchen so, eigene Deutungen zu etablieren (grundlegend Merten und Westerbarkey 1994; Saxer 2008). Zu beachten ist ferner, dass Deutungen nicht schlicht durchgesetzt werden können, sondern immer auch auf von anderen Akteuren eingebrachte Gegenentwürfe stoßen. Diese verschiedenen Deutungsangebote konkurrieren in der Folge um Aufmerksamkeit, und es liegt an der mobilisierbaren Durchsetzungsmacht, welches sich etabliert und höhere Reichweiten erzielt.

Als gutes Beispiel für diese Prozesse lässt sich das Ringen um eine sozialökologische Transformation der Gesellschaft verstehen, welches mit einem »Kampf um Aufmerksamkeit (Crary 2002; Franck 1998) und dominante Deutungshoheiten im öffentlichen Diskurs einhergeht. Neben inhaltlichen Kriterien (wie etwa Nachrichtenfaktoren) entscheiden auf einer vorgelagerten Ebene auch ökonomische Bedingungen und Strukturen darüber, welche Themen überhaupt in den Blick der Öffentlichkeit gelangen können (Herman und Chomsky 2002; Schade 2018). Diese Kriterien gelten als Ursachen für die auf den ersten Blick zu beobachtende Dominanz der Positionen ressourcenstarker Akteure in der Öffentlichkeit. Dagegen zeige ich im Folgenden, dass diese ökonomischen Mechanismen mitunter gezielt unterwandert werden - wie am Beispiel kleinerer sozialer Bewegungen zu erkennen ist.

Sozialen Bewegungen lässt sich gesellschaftlich die Rolle von Impulsgeberinnen zuschreiben: „Within the mediating sector of politics, social movements specifically serve to give the initial impulses 
for change (Neidhardt und Rucht 1991). Sie machen auf Missstände aufmerksam und wirken auf Veränderungen hin. Diese Akteure sind jedoch häufig weniger ressourcenstark und verfügen daher über weniger Möglichkeiten, Zugang zu relevanten Öffentlichkeiten zu finden (Schade 2018; Oy 1997). Folglich sind sie darauf angewiesen, sich auf unkonventionelle Weise Gehör zu verschaffen, und wählen subversivere und häufig subkulturell konnotierte Strategien zur Generierung von Aufmerksamkeit (Juris 2012; Ertl 2015; Veneti 2017). Diesbezüglich spreche ich im Folgenden von devianten Kommunikationsstrategien. Damit sind Strategien gemeint, die abweichendes Verhalten in Kauf nehmen oder aktiv suchen, um Aufmerksamkeit zu generieren. Fraglich ist in diesem Zusammenhang, ob Devianz neben der Aufmerksamkeit auch die Zustimmung für die vertretenen Botschaften und Deutungsangebote erhöht. Das heißt, deviante Kommunikationsstrategien bergen immer auch die Gefahr, die wahrgenommene Legitimität der Forderungen zu bedrohen, da die Form der Kommunikation als normverletzend und so als potenziell gemeinwohlschädlich abqualifiziert wird. Soziale Bewegungen müssen hier wesentliche Abwägungen treffen.

Die Forschung zur Devianz betrachtet bisher vorrangig Makro- und Mikrophänomene (Peuckert 2016; Lamnek 2013). Die Beschreibung einer Mesoperspektive, die deviantes Verhalten von organisationalen Akteuren in den Blick nimmt, ist bisher eher randständig. Zwar beschreibt etwa Luhmann (1999) nonkonformes Verhalten von Organisationsmitgliedern mit dem Konzept der »brauchbaren Illegalität«, geht damit jedoch auf organisationsinterne Normen ein. Nach außen beziehungsweise auf die Gesellschaft orientiertes Verhalten lässt sich dagegen ideal mit einer neo-institutionalistischen Perspektive auf Organisationen verbinden, welche gesellschaftliche Erwartungen an Organisationen in den Mittelpunkt rückt (Meyer und Rowan 1977; Sandhu 2012; Kette und Barnutz 2019). Auf diese Perspektive möchte ich im Folgenden zurückgreifen, um Verhaltenserwartungen an handelnde Personen auf gesellschaftliche Erwartungen an Organisationen zu übertragen.

In meinem Beitrag gehe ich folgendermaßen vor. Zuerst stelle ich das Verständnis von Organisation, Öffentlichkeit und Legitimität heraus, um dieses für die Kommunikation sozialer Bewegungen zu konkretisieren. Darauf aufbauend nehme ich auf das Konzept der Gegenöffentlichkeit (Ahlemeyer 1995; Scholl 2009; Wimmer 2007) Bezug, bevor ich im nächsten Schritt das Konstrukt der Devianz erläutere. Hier mache 
ich insbesondere von dem an sozialen Zuschreibungen orientierten »Labeling-Ansatz« (Lamnek 2013) Gebrauch. Nach der Begriffsklärung beziehe ich die Devianz auf die Strategische Kommunikation und Eindruckssteuerung (Ebert und Piwinger 2007) von Organisationen und sozialen Bewegungen und stelle hier Potenziale sowie Risiken heraus. Konkret illustriere ich schließlich am Fall der umstrittenen Rodungsarbeiten am Hambacher Forst, wie Devianz als analytische Kategorie bei der Untersuchung Strategischer Kommunikation behilflich sein kann. Mittels einer rekonstruktiven Bildanalyse (Przyborski und Slunecko 2012) der Kommunikation der Initiative Ende Gelände wird verdeutlicht, dass die Inszenierung funktionaler Devianz großes Potenzial für Legitimitätsgewinne bietet.

\section{Organisation, Öffentlichkeit und Legitimität}

Aus neo-institutionalistischer Perspektive kommt Organisationen der Status eines organisationalen Akteurs zu. Das bedeutet, dass Organisationen nicht nur handlungsfähig, sondern auch aktiv und interessengetrieben sind (Meyer und Rowan 1977). Sie sehen sich in einer komplexen Gesellschaft mit diversen und teils disparaten Erwartungen aus der Umwelt konfrontiert, mit denen sie umgehen müssen (ebd.; Sewell 1992). Diese Erwartungen werden als sozial etablierte Muster und Schemata - als Institutionen - gefasst (Sandhu 2012; Sewell 1992). Idealerweise sind die an die Organisation gestellten Erwartungen dieser dienlich und ihren Handlungen wird von den für sie relevanten Öffentlichkeiten Legitimität zugeschrieben (Werder et al. 2018).

\subsection{Strategische Kommunikation}

Jegliche Handlung einer Organisation trägt zur Eindrucksbildung in spezifischen Öffentlichkeiten bei. Sind sie jedoch bestrebt, das Bild, welches ihre Umwelt von ihnen hat, und die damit verbundenen Erwartungen gezielt und organisationsdienlich zu gestalten, nutzen Organisationen Strategische Kommunikation (ebd.). Hierbei zeigt sich ein durchaus kreativer und strategischer Umgang mit den Anforderungen der Umwelt: Zwar wäre eine Anpassung der Organisationen an die Erwartungen denkbar (Isomorphie), eine solche gestaltet sich allerdings aufgrund deren disparater Natur schwierig bis unmöglich. Gängiger ist 
daher das zeremonielle Darstellen von Adäquanz durch organisationale Strukturen oder Rituale als »Legitimitätsfassade« (Sandhu 2012), um sich ein Stück weit von den für sie dysfunktionalen Erwartungen zu entkoppeln. Hierzu betreibt die Organisation auf der "Schauseite (Kühl 2011) gezielte Eindrucks- und Erwartungssteuerung oder »Impression Management « (Ebert und Piwinger 2007).

Nach Femers (2015) ist eine persuasive Eindruckssteuerung dann besonders erfolgreich, wenn die Organisation »Inszenierungen zeigt, die sich neben dem eigenen Wunschbild auch an gesellschaftlich relevanten Werten orientieren «. Das bedeutet, dass sie sich neben ihren funktionalen Kerndimensionen (wie zum Beispiel ökonomische Leistungsfähigkeit) auch darum bemüht, sich als gesellschaftlich wertvoll und als guter »Corporate Citizen« (Valor 2005; Kim und Lee 2018) darzustellen. Die Organisation betont somit Rechtschaffenheit und einen positiven gesellschaftlichen Beitrag.

Die zentralen Erwartungen, an denen sich eine Organisation ausrichtet, sind nicht statisch und je nach Zweck, den sie verfolgt, verschieden. So ist bei der Zuschreibung von Legitimität vorrangig die Einschätzung spezifischer Öffentlichkeiten, die der sogenannten Stakeholder*innen (Freeman 1984; Karmasin und Weder 2014), von Belang. Diesen wird organisationsseitig das Potenzial zugeschrieben, Handlungen positiv oder negativ zu beeinflussen. Als wichtige Stakeholder*innen sind vorab schon politische Entscheidungsträger*innen zu nennen, da sie für gesellschaftlich relevante Themen Regelungen anstoßen und den regulativen Rahmen für die Handlungen organisationaler Akteure bereitstellen (Melenhorst 2015). Wichtig ist an dieser Stelle zu verstehen, dass die zur Schau gestellten Werte je nach aktuellem Kontext, Organisationszweck und Stakeholder*innen-Kreis variieren, wie im Beispiel noch genauer zu sehen sein wird.

\section{2 »Klassische« Organisationen und Öffentlichkeit}

Medien kommt durch ihre Thematisierungsfunktion und die Herstellung von Öffentlichkeit (an der sich auch Politik orientiert) entsprechend eine besondere Bedeutung zu (Vliegenhart et al. 2016; Walgrave und van Aelst 2006), denn sie stellen ein »Netzwerk der gegenseitigen Beobachtung (Sandhu 2012, 106) zur Verfügung. Für Organisationen sind hier besonders Agenda- und Frame-Building-Effekte relevant (Buchmeier 2020; Colistra 2018; Hänggli 2012). Orga- 
nisationen achten folglich darauf, Themen, die deswegen für sie von Bedeutung sind, weil sie mit spezifischen Lösungen oder Positionen auf diese reagieren und sie zur strategischen Positionierung nutzen können, in den medialen Diskurs zu erheben und spezifisch zu framen. Das Gleiche gilt für die Kehrseite: Im sogenannten »Agenda Cutting « werden potenziell organisationsbedrohende Themen eben nicht thematisiert (Buchmeier 2020).

Wie bereits erwähnt, richten Organisationen ihre kommunikativen Bemühungen besonders an ihren spezifischen Öffentlichkeiten aus. Mittelbar ist jedoch auch eine eher disperse, allgemeinere und bürgerlicher geprägte Öffentlichkeit bedeutsam - der sogenannte »Mainstream« - (Scholl 2009, 84), da hier allgemeinere Erwartungen ausgehandelt werden und sich somit potenziell neue Themen und Ansprüche formieren können, auf welche die Organisation gegebenenfalls reagieren muss (Lauzen 1997). Sie adressiert daher nicht nur ihre unmittelbaren Stakeholder*innen, sondern ist auch an einer dienlichen Konstruktion der medialen Wirklichkeit (Merten 2009) durch aktive Beobachtung und Gestaltung von Themen in einer übergeordneten Öffentlichkeit - dem Mainstream - interessiert.

\section{Mainstream und Gegenöffentlichkeit}

Der Mainstream lässt sich grob als Ort der Bildung und Reflexion konsensueller Positionen beschreiben, in welchem gesellschaftlich zentrale Sinnmuster, Werte und Ideologien ausgehandelt, bestärkt und eine hegemoniale öffentliche Meinung etabliert wird. Dieser Prozess verstärkt sich durch eine medienübergreifende Konsonanzbildung zusätzlich (Krüger 2016). In der Bewegungsforschung wird die bürgerliche Öffentlichkeit als "gehobene, herrschende Mainstreamkultur« (Scholl 2009; Jacke 2004) thematisiert, an der sich eine »Gegenöffentlichkeit« reibt, um mit anderen Selektionen und Frames als im Mainstream eine kontrastierende Position einnehmen zu können.

Journalistischen Medien kommt neben der Konstruktion gesellschaftlicher Wirklichkeit eine diskursordnende Sonderrolle zu, in der den strategischen Selbstbeschreibungen der Organisationen kontrastierende Fremdbeschreibungen gegenübergestellt werden (Hoffjann 2011). Idealisierend wird eine grundlegend kritische Haltung des Journalismus gegenüber den strategischen Bestrebungen von machtvollen Akteuren unterstellt (Herman und Chomsky 2002). In der Praxis zeigt sich jedoch eine durchaus kooperative Zusammenarbeit organisationaler Pressestellen mit dem Journalismus (Altmeppen, Röttger und 
Bentele 2004; Macnamara 2014; Baerns 1991). ${ }^{1}$ Je ressourcenstärker die Akteure, je intensiver die organisationalen Kontakte in den Journalismus und je professioneller die Aufbereitung strategischer Kommunikationsmaterialien, desto größer ist empirisch die Übernahmewahrscheinlichkeit der vorbereiteten Inhalte und Botschaften sowie eine durch journalistische Medien legitimierte Positionierung in einer größeren Öffentlichkeit (ebd.). ${ }^{2}$ Für diese professionalisierte Kommunikation bedarf es Ressourcen, die in eine persuasive Inszenierung fließen und den Zugang zu Journalismus ermöglichen (Herman und Chomsky 2002). Mit Gamson und Wolfsfeld lässt sich ergänzen: »Organization, resources, and media sophistication are all markers journalists use to identify who the serious players are." (Gamson und Wolfsfeld 1993, 121; zitiert nach Schade 2018, 251) Und dies sind Merkmale, über die nicht alle Akteure gleichermaßen verfügen.

\subsection{Soziale Bewegungen als besondere Organisationen}

In der Konzeption als Organisationen stellen soziale Bewegungen eine Sonderform dar. Sie sind loser gekoppelt und hinsichtlich ihrer Eintrittsvoraussetzungen in der Regel deutlich geringer formalisiert als dies bei klassischen Organisationen wie Unternehmen oder Vereinen der Fall ist (Ahlemeyer 1995; Stickler 2005). Jedoch kommen soziale Bewegungen für ihre kollektiven Aktionen nicht ohne eine gewisse Stabilität und Grundstruktur aus, welche vorrangig durch eine »relativ stabile [Kern] Gruppe getragen werden« (Rucht 1984, 610).

Die Struktur einer Bewegung ist dabei abhängig von ihrer Zielsetzung. Rucht (ebd.; 1994) unterscheidet zwischen inward- und outward-orientierten Ausprägungen. Erstere wollen vorrangig das Verhalten ihrer Mitglieder ändern, während letztere auf »die Gesellschaft«

1 Aktuelle Studien zum Verhältnis zwischen Journalismus und PR gibt es nach Kenntnis der Autorin vorrangig von Praktiker*innen und Berufsverbänden. Hier sei beispielsweise auf Hadwiger (2019) verwiesen. Auskunft über wissenschaftliche Gütekriterien lassen sich aus diesen Publikationen häufig nicht entnehmen. Die Ergebnisse Hadwigers in einer Praktiker*innenbefragung in Journalismus und PR verweisen jedoch auf ein kooperatives Zusammenwachsen der beiden Disziplinen auf operativer Ebene.

2 Hier sei angemerkt, dass die Übernahmewahrscheinlichkeit in Krisenberichterstattung deutlich sinkt und stattdessen die journalistische Recherchetätigkeit zu diesen fraglichen Themen ansteigt (vgl. Brodbeck 2018, 287). 
oder zumindest so viele Menschen wie möglich abzielen. Daher werden sie auch als »machtorientierte Bewegungen« (Rucht 1984, 610) beschrieben. Sie tendieren zu stärker formalisierten Organisationen, da hier ein gewisser Grad an Planbarkeit und Routinisierung von Abläufen als notwendig unterstellt wird.

Wer zu einer Bewegung gehört und wer nicht, ist aushandlungsbedürftig, da formale Beitrittsroutinen wie bei anderen Organisationsformen entfallen. Strukturell beschreibt Rucht (ebd.) die Organisation sozialer Bewegungen deshalb mit Hilfe dreier konzentrischer Kreise. Im Zentrum steht für ihn die Kerngruppe, beziehungsweise die »Eliten«, die über mehr oder minder bindende Entscheidungskompetenzen verfügen. Darum gruppieren sich die »Basisaktivisten«, während sich am Rande Anhänger*innen und Unterstützer*innen sammeln (ebd., 612).

Die Funktion sozialer Bewegungen liegt in der Interessenartikulation und der Mobilisierung, welche sich auch als ihre Elementaroperation verstehen lässt (Ahlemeyer 1995; Rucht 1984; 1994). Luhmann (1994) verweist auf Protest als zentrale Funktion und Kommunikationsform. Hierbei versuchen soziale Bewegungen »Themen für politisch relevant zu erklären « (Jarren und Donges 2011, 130) und darauf bezogene transformative Handlungen hervorzurufen. Scholl $(2009,85)$ beschreibt die Funktion auch als das Aufzeigen von Alternativen »zur aktuellen politischen, ökonomischen, rechtlichen usw. Verfasstheit der Gesellschaft allgemein sowie zu den politischen, ökonomischen, rechtlichen usw. Organisationen und partikularen Handlungen speziell«. Soziale Bewegungen lassen sich also auch als Gegenentwurfsakteure beschreiben.

Im Folgenden werde ich mich auf die kommunikativen Handlungen und Strukturen von machtorientierten Bewegungen konzentrieren. Diese sind zur Erreichung ihrer Ziele auf öffentliche Aufmerksamkeit angewiesen - und entsprechend auch auf Strategische Kommunikation.

\subsection{Soziale Bewegungen und Öffentlichkeit}

Scholl (2009) verortet die Kommunikation sozialer Bewegungen vorranging im Spektrum sogenannter Alternativmedien. Diese lassen sich mit Bezug zu Rucht (1994) und Oy (1997) als Ort eigener Themensetzungen konzipieren, die im sogenannten Mainstream nicht oder nur am Rand auftauchen. Da Akteure hier zunächst weitestgehend Deutungshoheit über die von ihnen publizierten Botschaften haben, sind sie vorrangig als Ort der internen Selbstvergewisserung zu denken, die 
eine »innere Mobilisierung « der Bewegungsmitglieder gegen vielfältige Positionen des Mainstreams ermöglicht. Auch teilöffentliche Foren und Chaträume in Sozialen Medien lassen sich zu diesen eher internen Räumen zählen (Harlow 2012; Kidd und McIntosh 2016).

Um jedoch auch in der bürgerlichen Öffentlichkeit Aufmerksamkeit zu erlangen, sind soziale Bewegungen auf die Thematisierung ihrer Deutungsangebote im Mainstream angewiesen: a) um bei Bürger*innen Aufmerksamkeit für ihre Anliegen zu erzeugen, b) um ihre Anliegen als legitime Forderungen positionieren zu können, c) um für diese Anliegen weitere potenzielle Unterstützer*innen zu gewinnen und d) um vermittelt durch eine breitere Medienöffentlichkeit »auf politische Entscheidungsträger einzuwirken« (Rucht 1994, 348).

Schade $(2018,254)$ benennt Publizität für soziale Bewegungen als oberste Priorität:

Wenn [soziale Bewegungen] ihre Anliegen öffentlich machen, »können sich die Eliten nur bei Strafe des Legitimitätsverlustes einer Auseinandersetzung mit ihnen verweigern." (Schmitt-Beck 1998, 21). Denn dadurch wird der von einer Bewegung angemeldete Entscheidungsbedarf als legitim anerkannt und mit hoher Wahrscheinlichkeit in Entscheidungsprozesse eingespeist (auf die politische Agenda gesetzt). (Schade 2018, 254)

Diese Argumentation erscheint ad hoc plausibel. Allerdings lässt sie den Modus der Auseinandersetzung beziehungsweise der Entscheidung außer Acht und setzt Publizität mit Legitimität gleich. So ist zu bedenken, dass die öffentliche Aufmerksamkeit zwar zu einer Auseinandersetzung mit einer Bewegung zwingt. Eine inhaltliche Auseinandersetzung mit den Positionen, die diese Bewegung für gesellschaftlich relevant erklären möchte, ergibt sich daraus jedoch nicht zwingend. Je nach Kontextualisierung und gewähltem Framing in der Berichterstattung können auch andere Aspekte ins Zentrum der öffentlichen Debatte gerückt werden (Ertl 2015; Hänggli 2012). Dementsprechend ist eine Reaktion auf mehreren Ebenen möglich, die eine Auseinandersetzung mit den inhaltlichen Forderungen der Bewegung sogar explizit vermeidet. $\mathrm{Zu}$ diesen zählt einerseits die Akteursebene: Eine Strategie zum Umgehen der inhaltlichen Auseinandersetzung ist die Personalisierung. Statt über die bewegungsseitig angebrachte Kritik und Veränderungspotenziale zu sprechen, werden die handelnden Akteur*innen (ab-)qualifiziert und ihr Anliegen dadurch delegitimiert. Unrühmliches Beispiel ist hier die Klimaaktivistin Greta Thunberg, die sich persönlichen Angriffen in Bezug auf ihr Geschlecht, ihr Alter oder das Asperger- 
Syndrom ausgesetzt sieht (Möllers 2019). Auf der Formebene konzentriert sich die Auseinandersetzung hingegen auf die äußere Form der Mitteilung oder des Protestes, indem deren Angemessenheit als fraglich gekennzeichnet wird. Beispielhaft sind hier die Interventionen des Zentrums für politische Schönheit zu nennen (Gerk und Bernhard 2019) oder Protestaktionen verschiedener Umwelt- und Klimaaktivist*innen (Ehl und Hänlein 2019).

Ertl $(2015,147)$ stellt heraus, »dass Proteste im Laufe der Jahre zunehmend als Ärgernis geframed werden [und] vielmehr als die Forderungen des und die Gründe für den Protest Taktik, Spektakel und dramatische Aktionen medial veröffentlicht [werden] «. Hierzu verweist sie auf die empirischen Arbeiten von Harlow und Johnson (2011), Boyle, McLeod und Armstrong (2012) sowie Di Cicco (2010). Ertl (2015, 146-147) führt des Weiteren aus: »Extremeく und radikale Protestkollektive, die am Status quo rütteln, rufen eher eine negative, marginalisierende Medienreflexion bzw. Berichterstattung hervor.« Teune und Sommer (2017, 30-32) differenzieren in einer Analyse der deutschen Medienberichterstattung zu Protesten, dass insbesondere »konservative « Medien in ihrer Berichterstattung weniger die inhaltliche Kritik in den Fokus rücken, sondern die Form des Protests. Protestierende selbst kommen zudem seltener selbst zu Wort. Kommt es bei Protesten »zu Konfrontationen mit der Polizei« (ebd., 26), sinkt in allen Medien die Auseinandersetzung mit den Hintergründen und die Beschreibung der normverletzenden, also devianten Handlungen und der Reaktionen darauf dominiert. Spektakel und potenzielle Normverletzungen, die von sozialen Bewegungen ausgehen, liefern also Stoff für Berichterstattung und damit für Publizität - jedoch auch Potenzial für den Wechsel der Thematisierungsebene. Das Protesthandeln wird so zwar publik, erfährt aber eine Rahmung, die von Irritation bis hin zu Kriminalität reicht. Es bedient dominante Nachrichtenfaktoren wie Konflikt und Gewalt (Bonfadelli und Friemel 2001; Schulz 1979), die in journalistischer Selektion und (notwendiger) Verkürzung in den Vordergrund rücken, die inhaltlichen Positionen jedoch überlagern.

Dazu kommt, dass, wie eingangs dieses Abschnitts skizziert, soziale Bewegungen nicht zu klassischen Formaten der Strategischen Kommunikation neigen (Oy 1997). Dies kann bedingt sein durch ihre Struktur und ihren Ressourcenmangel, aber auch durch die subkulturelle Prägung, die klassische Formate der Medienarbeit quasi unmöglich machen (Veneti 2017; Philipps 2012). Gemeint sind damit Deutungen, kulturelle Praktiken, aber auch eine eigene Protestästhetik (Veneti 
2017), die spezifische subkulturelle Codes und Identifikationsmodelle hervorbringen (Oy 1997, 79; Lamnek 2013). Die in diesen Kontexten ausgebildeten Anerkennungssysteme können beinhalten, dass Handlungen und Ziele goutiert werden, die außerhalb dieses Raumes keine Anerkennung finden (Lamnek 2013, 185-187). Gerade vor dem Hintergrund der sogenannten bürgerlichen Öffentlichkeit haben beispielsweise das Ausüben von Gewalt oder das unerlaubte Eindringen in privates Gelände das Potenzial, normverletzende wenn nicht gar kriminelle Zuschreibungen hervorzurufen. In klassischen Medienformaten wird es deshalb schwierig, die eigenen inhaltlichen Positionen unterzubringen.

Indes lässt sich argumentieren, dass soziale Bewegungen für tatsächlichen gesellschaftlichen Wandel auf Anerkennung und Zuschreibung von Legitimität aus dem bürgerlichen Mainstream, den sie mitunter ablehnen und in dem sie als deviant geframed werden, angewiesen sind. Mithin stecken kleinere soziale Bewegungen in einem ressourcen- und kulturbedingten Dilemma: Um gesellschaftliche Transformation und im Wortsinn »Bewegung « zu erreichen, sind sie auf Aufmerksamkeit für ihre Positionen angewiesen, die über die eigene Bezugsgruppe hinausgeht. Die These im Folgenden wird deshalb sein: Da sie in einem auch an sozialen und ökonomischen Ressourcen orientierten Mediensystem einen schweren Stand haben, setzen kleinere soziale Bewegungen zwar auf spektakuläre und "photografierbare (Luhmann 1991, 152) Aktionen, um Aufmerksamkeit zu erlangen. Diese bergen jedoch immer das Risiko, als Normverstöße in den Fokus zu rücken und damit vom eigentlichen inhaltlichen Anliegen abzulenken. Das leitet über zum Konzept der Devianz.

\section{Devianz und die Inszenierung von Protest}

Dass soziales Verhalten an Normen gebunden ist, »ist eine einfache, ja triviale Alltagserfahrung « (Popitz 1961, 185). Normen dienen dazu, soziales Verhalten in einem gewissen Maße zu konditionieren und erwartbar zu machen. Devianz stellt die Abweichung von diesen Erwartungen dar. Krumpal und Berger $(2020,1)$ fassen darunter »Verhaltensweisen oder Einstellungen [...], welche von allgemein anerkannten, normativen Erwartungen einer Gesellschaft abweichen und entsprechend einer informellen oder formellen Sanktionsdrohung unterliegen«. Je nach Kontext und Grad der Normverletzung kann das Sanktionspotenzial 
von sozialer Ächtung bis hin zu gerichtlicher Ahndung reichen. Zweck der Kennzeichnung, Ächtung und Sanktionierung von Devianz ist die Durchsetzung einer Normierung in bestimmten sozialen Kontexten:

Gemeinsam ist allen Arten abweichenden Verhaltens, dass es sich um Abweichungen von gesamtgesellschaftlich dominanten Normen handelt, die jeden Menschen als Mitglied der Gesellschaft betreffen und der Stabilisierung des gesellschaftlichen Status quo - nicht nur in Form gesetzlicher Regelungen, sondern auch in Form bestimmter Leitbilder von "geordneten « Verhältnissen in den zentralen gesellschaftlichen Bereichen Arbeit, Konsum und Familie - dienen. (Peuckert 2016, 129).

In dieser Ausarbeitung soll es um den spezifischen Prozess der Rahmung eines Verhaltens als deviant und unangemessen gehen. Im Folgenden werde ich daher insbesondere auf den »Labelling-Ansatz« der Devianz eingehen.

Dieser geht davon aus, dass eine Abweichung von einer Norm als "Zuschreibungsprozess des Attributes der Devianz zu bestimmten Verhaltensweisen im Rahmen von Interaktionen « (Lamnek 2013, 223) zu verstehen ist. Ob ein Verhalten also überhaupt als deviant wahrgenommen wird, ist nicht von vorneherein auszumachen. Becker (2014, 31) stellt heraus, »dass gesellschaftliche Gruppen abweichendes Verhalten dadurch schaffen, dass sie Regeln aufstellen, deren Verletzung abweichendes Verhalten konstituiert, und dass sie diese Regeln auf bestimmte Menschen anwenden, die sie als Außenseiter etikettieren«. Die Frage, ob etwas von einer Norm abweicht oder nicht, wird somit als aushandlungsbedürftig sowie kontext- und personenabhängig angesehen (Lamnek 2013, 223). Und so haben »einige Abweichler [...] Mitleid zu erwarten, andere verbreiten Angst oder werden verachtet« (Peuckert 2016, 129).

Abweichendes Verhalten ist demnach nicht objektiv zu identifizieren und keine Qualität, die einer Handlung inhärent wäre, sondern wird vielmehr durch gleichzeitige Normsetzung definiert. Mithin ist die Markierung einer Abweichung ein alltäglicher, unausweichlicher Vorgang. Gleichwohl ist auf selektive Normanwendungen hinzuweisen: Gleiche Verhaltensweisen werden mitunter situations- und personenspezifisch unterschiedlich definiert (Lamnek 1977, 77-78; 2013). Je nach Adressat*in kann ein Verhalten als konform oder deviant gelten:

Da allgemeine Normen immer auf bestimmte Situationen bezogen werden müssen und somit interpretationsbedürftig sind, entscheidet das soziale 
Publikum darüber, ob einer bestimmten Handlung oder Person das Etikett abweichend zugeschrieben wird oder nicht. (Peuckert 2016, 141)

Bezogen auf das hier behandelte Thema heißt das, dass (kleinere) soziale Bewegungen und ihr Handeln von diversen medialen Publika unterschiedlich bewertet werden können. In Bezug auf das skizzierte Mediendilemma sozialer Bewegungen ist dabei zu beachten, dass »klassischen « Medien eine wesentlich höhere Reichweite im sogenannten Mainstream und damit Deutungsmacht in der Zuschreibung von Devianz zukommt (Hänggli 2012).

\section{Funktionale und dysfunktionale Devianz}

Wie zuvor erläutert, dient das Sanktionspotenzial hinter der Normierung von Verhalten dem Erhalt sozialer Ordnung. Zentral ist jedoch auch, dass sich diese Ordnung immer wieder aufs Neue bewähren muss, also fortlaufend auch in Frage gestellt wird. Durch diese Aushandlungs- oder Vergewisserungsprozesse, durch die geltende Regeln und Normen auf ihre Legitimität hin überprüft werden, kann sich eine Gesellschaft erneuern (Lamnek 2013, 45). Die Abweichung von der Norm erscheint so als »funktionale Devianz« (Peuckert 2016, 132). Nimmt die Abweichung allerdings überhand, kann sie eine Gesellschaft auch durch Gewalt und Kriminalität zutiefst schädigen. Diese Fälle von dysfunktionaler Devianz haben eher soziale Desintegration der Akteur*innen und deren Anliegen zur Folge.

Protest richtet sich immer gegen herrschende Normen und hat die Einführung einer neuen Norm zum Ziel (Scholl 2009). Dies birgt zunächst großes Irritationspotenzial für die herrschende Mehrheit. Entsprechend hoch ist der Nachrichtenwert, wenn Konflikt und Rebellion, Neu- und Andersartigkeit zusammenkommen. Abweichungen von einer bestehenden Norm weisen immer auch auf Grenzüberschreitendes bis hin zum Skandalösen hin und bedienen so journalistische Selektionskriterien. Gleichzeitig schlägt eine gesamtgesellschaftliche Ablehnung devianten Verhaltens zu Buche und ein stärker ausgeprägtes Konformitätsstreben der »Mitte«, die sich mit dem Mainstream und seinen Positionen identifiziert. In bestimmten Situationen kann es jedoch auch zu einer breiten Solidarisierung mit dem »Underdog « kommen (Menzel 1997, 119). Nämlich insbesondere dann, wenn die Devianz als funktional und innovativ für die Mehrheitsgesellschaft erkannt wird. Diese Zuschreibung birgt großes Potenzial für die Strategische Kommunikation sozialer Bewegung und die Inszenierung von Protest, wie ich am folgenden Fallbeispiel deutlich machen möchte. 


\section{$4 \quad$ Fallbeispiel Hambacher Forst}

Der Hambacher Forst ist ein etwa 500 Hektar großes Waldgebiet zwischen Köln und Aachen. Ebenso ist er Schauplatz diverser Interaktionen zwischen aktivistischen Protestformen und staatlichen Eingriffen. In diesem Abschnitt werden nun die dabei entstandenen Konflikte der Jahre 2017 bis 2019 als Fallbeispiel für die Strategische Kommunikation sozialer Bewegungen herangezogen. Im beschriebenen Fall gelang es diesen nämlich äußerst gut, hohe gesamtgesellschaftliche Anschlussfähigkeit herzustellen.

Bereits seit den 1970er Jahren rodet der Energiekonzern RWE den Hambacher Forst zur Braunkohlegewinnung. Dagegen hatte sich vor Ort schon gegen Ende des Jahrzehnts eine aktive Widerstandsbewegung gebildet. Diese intensivierte sich in den 2010er Jahren und errichtete an mehreren Stellen im Wald Barrikaden und Protestcamps, die immer wieder gewaltvoll unter großem medialem Interesse geräumt, aber stets wiederaufgebaut wurden (Dalkowski 2018).

2018 beschloss RWE weitere großflächige Rodungsarbeiten im Hambacher Forst. Eine Klage des BUND gegen diese Rodung wurde abgewiesen. Dies hatte eine erneute Räumung der Protestcamps zur Folge, die jedoch zu erheblichen Auseinandersetzungen zwischen Polizei und Umweltaktivist*innen führte. Nicht zuletzt deshalb richtete sich das öffentliche Interesse auf die Geschehnisse im Hambacher Forst, der bereits zuvor zum Symbol der Anti-Kohle-Bewegung geworden war. Zentral in diesem Protest waren die Initiativen »Hambi bleibt« und »Ende Gelände«. Ihnen gelang es, einer devianten linken Protesterzählung einen zunehmend gesellschaftlich anschlussfähigeren David-gegen-Goliath-Topos entgegenzustellen. Eine Großdemonstration mit rund 50000 Teilnehmer*innen am Hambacher Forst stand exemplarisch für die hohe Anschlussfähigkeit ihrer Botschaften. Den Initiativen gelang es, Devianz und die damit verbundene Aufmerksamkeit strategisch zu kapern, ein funktionales Narrativ zu etablieren und somit Reichweite und Zuspruch zu generieren. Zum Vergleich werden nun ein Pressebild der dpa und ein PR-Bild der Initiative »Ende Gelände« in einer rekonstruktiven Bildanalyse (Przyborski und Slunecko 2012) gegenübergestellt, um den Unterschied zwischen der Selbst- und der Fremddarstellung hinsichtlich der gezeigten Devianz zu verdeutlichen. 


\subsection{Rekonstruktive Bildanalyse}

Neben sprachlichen Botschaften aller Art erfahren visuelle Botschaften insbesondere durch die Dominanz Sozialer Medien wie Facebook, YouTube und Instagram zunehmend Beachtung in der Strategischen Kommunikation (Goransson und Fagerholm 2018). Doch auch analytisch ist ein »visual turn « (Burri 2008) in den Sozialwissenschaften zu konstatieren. Bilder lassen sich als Artefakte visueller Kulturen interpretieren und verweisen auf gestaltete Konstruktionen von Realität und Bedeutung (Phillips 2012, 2; Przyborsky und Slunecko 2012). Vor diesem Hintergrund ziehe ich im Folgenden ein Bild einer Pressepublikation heran und stelle es einem Bild, das von »Ende Gelände « publiziert wurde, gegenüber. ${ }^{3}$ Beide stellen freilich nur einen Ausschnitt des Geschehens dar. Die Analyse soll aber vorrangig verdeutlichen, wie unterschiedlich und geradezu strategisch Devianz aufgegriffen werden kann. Im Folgenden wird eine rekonstruktive Bildanalyse nach Przyborsky und Slunecko (2012; grundlegend Bohnsack 2009; Panofsky 1975) durchgeführt. In dieser wird zunächst auf den generellen Bildaufbau und dann auf die Ikonologie eingegangen.

\subsection{Bildanalyse 1}

Das erste Bild (Abb. 1) zeigt eine Aufnahme aus dem besetzten Hambacher Forst. Im Zuge eines tödlichen Sturzes eines Journalisten war die Räumung im Forst vorübergehend ausgesetzt worden. Durch die gewählte Perspektive des Bildes empfinden sich die Betrachtenden als Teil des Geschehens. Die Bildtemperatur ist eher kühl und die Farben sind dunkel und getrübt; das Bild wirkt nass.

Die dominanteste Linie stellt eine Barrikade aus zusammengetragenem (Sperr-)Holz und Reisig dar, die das Bild in einer absteigenden Linie nahezu in der Mitte teilt. Sie wirkt behelfsmäßig, aber gerade durch die beiden Baumstämme massiv. Für ihre Konstruktion wurde vor allem natürliches, urwüchsiges Material verwendet. Es ist keine technisch hochwertige, militärische Barrikade, sondern ein chaotischer Haufen.

3 Die Analyse wurde mit der Unterstützung von Studierenden der Hochschule Osnabrück im Seminar »Übung Kommunikationskonzept« in mehreren Iterationen durchgeführt. An dieser Stelle möchte ich mich für die angeregte Diskussion bedanken. 
Gleichzeitig weckt sie auch Assoziationen mit einem Abriss oder einem behelfsmäßig errichteten Scheiterhaufen. Mit der Barrikade verwoben ist ein Protestschild mit der Aufschrift »Ende Gelände. Der $\mathrm{CO}_{2}$-Countdown läuft.« Das Schild wirkt ausrangiert und auf den Müll geworfen. Der Kampf um den Wald scheint verloren.

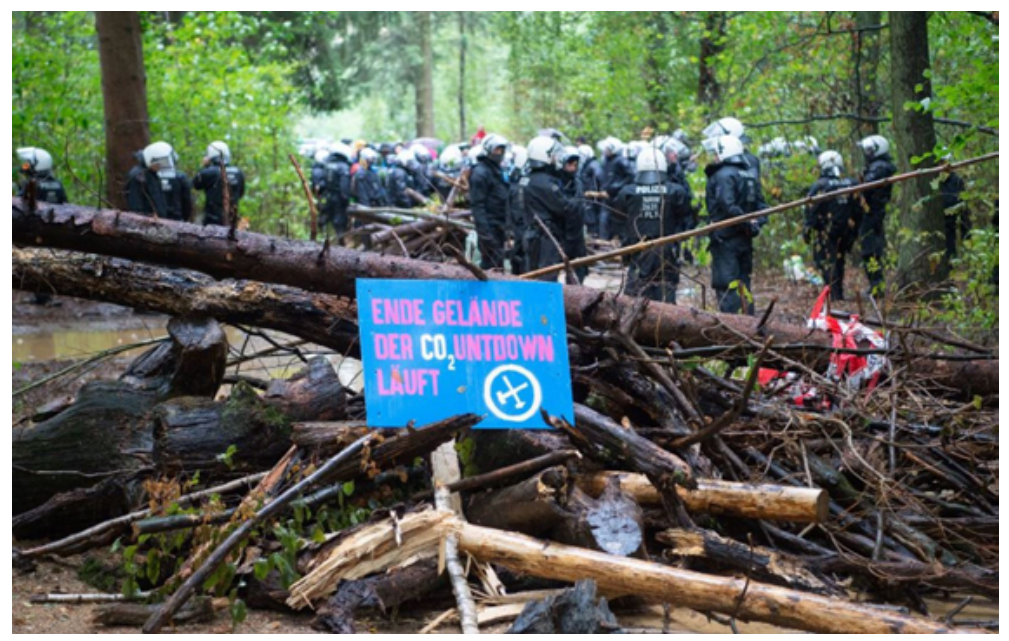

Abb. 1: dpa-Bild zur Dokumentation der Proteste im Hambacher Forst der Organisation »Ende Gelände« (Gateau, 24.09.2018).

Die Barrikade teilt das Bild und den abgebildeten Raum in ein Davor und ein Dahinter. Im Hintergrund, mit verminderter Schärfe, sind Polizist*innen in Einsatzkleidung zu sehen, welche mit der scheinbaren Natürlichkeit kontrastieren. Durch ihre Einsatzkleidung und Helme wirken sie nicht wie Personen, sondern wie die anonyme Exekutive, die deutlich besser ausgestattet erscheint als die auf dem Bild nicht sichtbaren Erbauer*innen der Barrikade.

Visuell wird eine klare Trennlinie zur Polizei als Repräsentation des Rechtsstaats und Vertretung von Recht und Ordnung gezogen. Die Barrikade mit Schild weckt Assoziationen an Elemente »klassischer « Protestästhetik wie brennende Straßenbarrikaden, die eine kritische Auseinandersetzung mit staatlicher Gewalt manifestieren. Damit ist bildsprachlich ein Moment der Devianz markiert, wobei die Polizei als Ordnungshüterin bereits vor Ort ist und die Lage beruhigt hat. Das Protestgeschehen bleibt unsichtbar und ist nur latent durch die zurückgelassene Barrikade im Bild enthalten. 


\subsection{Bildanalyse 2}

Das zweite Bild (Abb. 2) zeigt den Protest am Rande des Braunkohleabbaugebietes. Neben diversen Publikationen der Organisation »Ende Gelände « findet sich das Bild auf der Fotoplattform flickr in einem Album mit der Bezeichnung »Ende Gelände November 2017 - as it happened «. Bereits durch diese Bezeichnung wird ein Gegengewicht zu presseöffentlichen Darstellungen angedeutet (vgl. Abschnitt 2.3).

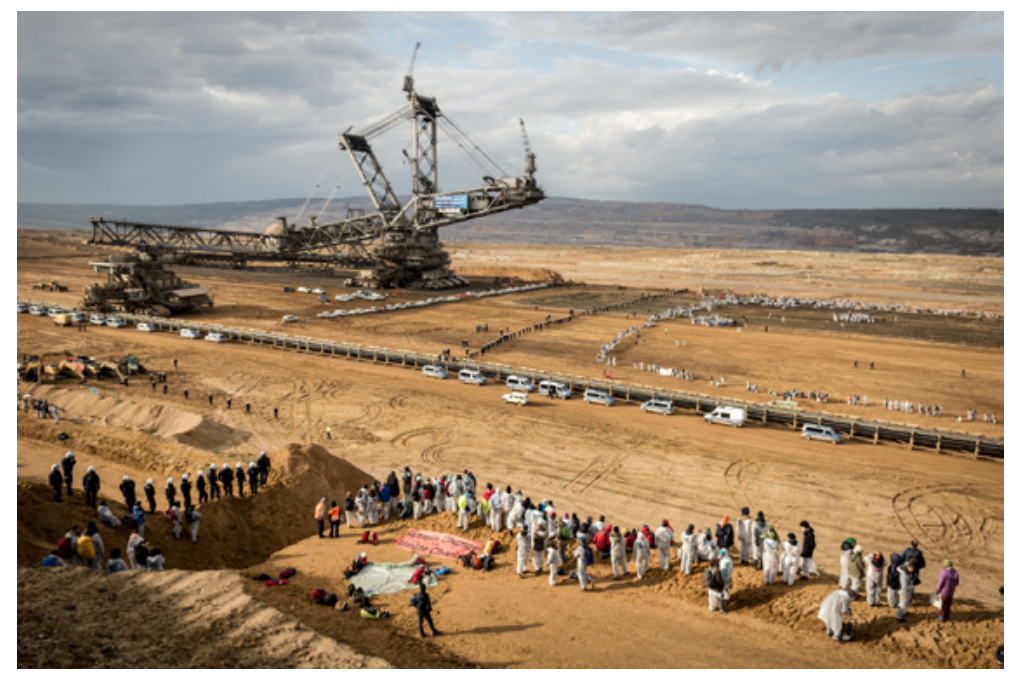

Abb. 2: PR-Bild der Organisation »Ende Gelände« (Bock, 05.11.2017).

Das Bild teilt sich in vier Bereiche. Im Vordergrund reihen sich Protestierende und Polizist*innen am Rande des Tagebaus auf, wo sich eine befestigte Absperrung in Form eines Zauns befindet. Zentral im Bild ist ein Bagger inmitten einer Kohlegrube zu sehen. Auch hiervor finden sich Protestierende und Polizei. Das vierte Element bildet der verblassende und verblauende Horizont. Farblich ist das Bild vor allem in Wüstentönen gehalten, die Assoziationen zu Trockenheit und Ödnis wecken. Aus dem Forst ist eine Kohlegrube geworden.

Die Polizist*innen im Vordergrund sind in ihrer Einsatzkleidung auch in diesem Bild nicht als Personen voneinander zu unterscheiden. Allerdings ist die Kluft der Protestierenden ebenfalls uniform, hier handelt es sich um Überzüge, wie sie für Malerarbeiten verwendet werden. In Verbindung mit dem öden und »toten« Hintergrund wirken sie jedoch 
wie Schutzanzüge, die bei Katastrophenfällen zum Einsatz kommen. Die Menschen müssen sich in und vor dieser lebensfeindlichen Umgebung schützen. Das Bild suggeriert dadurch einen Blick in eine dystopische Zukunft, die von profitgenerierender Maschinerie und nicht von und für Menschen gestaltet wird.

Im Zentrum des Bildes steht der Kohlebagger, der als Werkzeug der Organisation RWE die alte Industriemoderne repräsentiert. Dem gegenüber stehen die Menschen, die eine Abkehr von dieser Zeit und ihren Zielstellungen anstreben. Die Anordnung der Protestierenden und der Polizei erinnert an Schlachtreihen. Erstere sind in der Überzahl, jedoch ist ihre Formation weniger akkurat und organisiert als die der Polizei. Diese steht im Bild auf Seiten des Kohlebaggers und RWE - die Exekutive - erscheint als Verteidigerin der bestehenden Ordnung, die sich mit breiter Brust vor die Vergangenheit stellt und diese vor dem (unbewaffneten und weniger organisierten) Protest beschützt.

Die Zuschreibung von Devianz und Legitimität erfährt in diesem Bild gleichwohl eine Umkehr. Durch die verwendete Farbigkeit und Ödnis werden Endzeitszenarien wie im Film Mad Max aufgerufen, die einen Blick in eine potenzielle Zukunft gewähren. Das potenziell deviante Verhalten der Protestierenden und das zur Schau gestellte Machtverhältnis zur Polizei lässt sich im Bild als Aufstand für das Natürliche lesen, der eine herannahende Dystopie abwenden will. Der vermeintliche Angriff auf den Rechtsstaat und das unbefugte Betreten des Meilers werden nun durch die übergeordnete gute Sache der Daseinsvorsorge legitimiert, an der auch die Allgemeinheit Interesse hat. Entsprechend erfolgt die Abbildung der Normverletzung nunmehr als eine funktionale Devianz. Umgekehrt wird das bisher erwartungskonforme Verhalten von RWE (der Abbau von Braunkohle) als normverletzend inszeniert. Das Bild zielt darauf, das Festhalten am Status quo als potenziell gemeinwohlschädlich zu framen und zu delegitimieren.

\section{Strategisches Reframing}

Im zweiten Bild zeigt sich eine Abkehr von klassisch rebellischer und devianter Protestästhetik (Veneti 2017) hin zu einem stärkeren visuellen Verweis auf übergeordnete Topoi (hier beispielsweise David und Goliath). Der Bewegung gelingt in diesem Bild eine »Rahmenveränderung bzw. Neuinterpretation der Situation, die sog. Frame Transformation. [...] Ereignisse, die bereits in einem Rahmen interpretiert wurden, erhalten plötzlich eine andere, neue Bedeutung im Kontext eines neuen Rahmens, so dass sich die Wahrnehmung und Erfahrung der Situation än- 


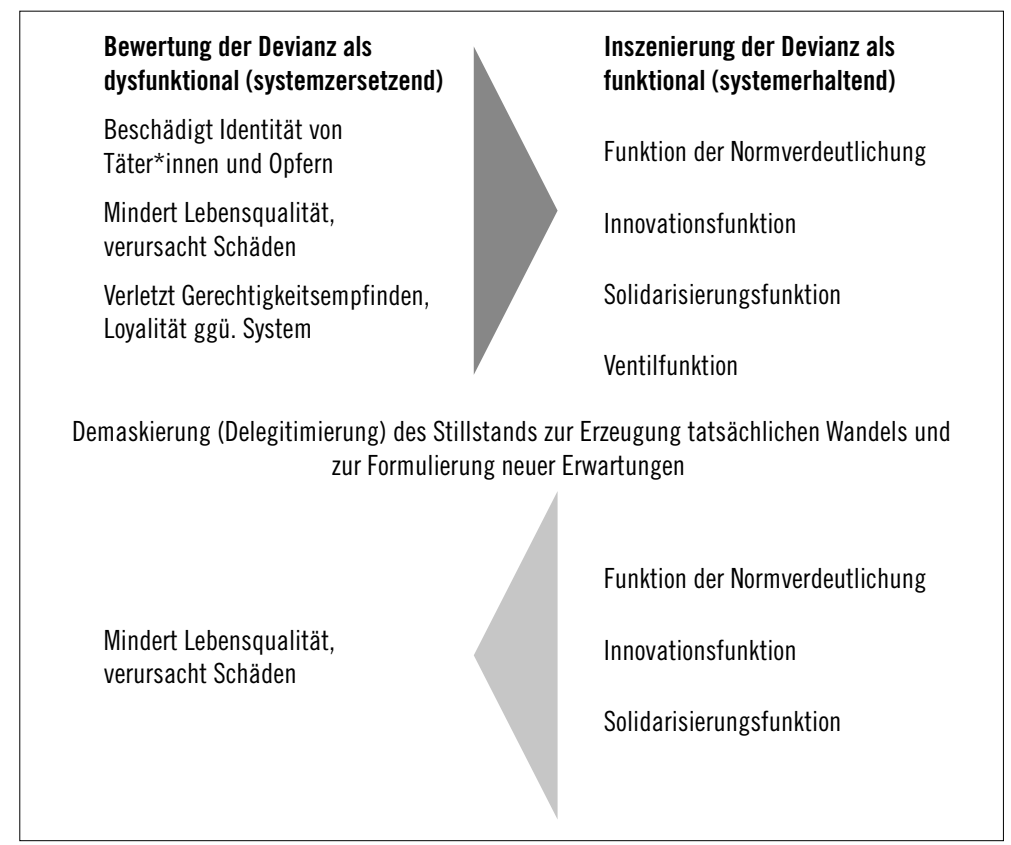

Abb. 3: Prozess des Reframings der Devianz-Zuschreibung (eigene Darstellung nach Peuckert 2016).

dert« (Schade 2018, 277; grundlegend hierzu Snow et al. 1986, 473-474). Schade verweist weiter darauf, dass insbesondere durch den Wandel des Frames bisher tolerierte Problemstellungen nun als nicht mehr hinnehmbar gesehen werden. Als besonders zielführend sieht sie hier die Umkehr der Schuldzuschreibung an, welche im zweiten Bild besonders im Mittelpunkt steht.

Anhand dieser Bilder zeigt sich, wie die Bewertung der Proteste im Hambacher Forst von ihrer ursprünglich systemzersetzenden Interpretation hin zu einer funktionalen und systemstärkenden Devianz umgedeutet und aufmerksamkeitsstark eingesetzt wurde (siehe Abschnitt 3). Im zweiten Bild ist eine klare Verschiebung in der Zuschreibung der dysfunktionalen Devianz erkennbar: Das Handeln der Organisation RWE, obwohl eigentlich formalrechtlich legitimiert, erfuhr Delegitimation in der öffentlichen Wahrnehmung, was zur Formulierung neuer Erwartungen führte (siehe Abschnitt 2). Nun ist nicht mehr die soziale Protestbewegung Träger der dysfunktionalen Devianz, sondern RWE, die durch das Festhalten an delegitimierten Normen systembe- 
drohende Muster reproduzieren. Der Prozess ist in Abb. 3 nochmals grafisch dargestellt.

\section{$5 \quad$ Zusammenfassung und Fazit}

In diesem Beitrag stand die Frage im Mittelpunkt, inwieweit der strategische Einsatz devianter Kommunikationsformen förderlich oder hinderlich für das Erreichen der Ziele einer Bewegung sein kann. Nach dem Darlegen der theoretischen Bausteine habe ich meine Überlegungen am Bespiel der visuellen Darstellung der Proteste im Hambacher Forst verdeutlicht.

Aufgrund von häufig geringerer Ressourcenausstattung haben es soziale Bewegungen nicht leicht, ihre Anliegen einer breiten Öffentlichkeit zugänglich zu machen. Sie sind daher auf aufmerksamkeitsstarke Ereignisse und sowohl die »Photografierbarkeit« (Luhmann 1991, 152) als auch zunehmend auf die Filmbarkeit ihrer kommunikativen Handlungen angewiesen. Ertl (2015, 78-80) beschreibt dies auch als die "Spektakularisierbarkeit« der Protestaktionen sozialer Bewegungen. Diese bedienen insbesondere die Nachrichtenfaktoren Konflikt, Schaden, Gewalt bis hin zur Kriminalität und auch das Aufbegehren gegen Eliten (Bonfadelli 2000; Schulz 1976). Sie sind damit hochgradig aufmerksamkeitsstark, jedoch auch hochgradig deviant.

Für tatsächlichen gesellschaftlichen Wandel sind soziale Bewegungen auf Aufmerksamkeit und eine gewisse Zuschreibung von Legitimität seitens einer bürgerlichen Öffentlichkeit angewiesen, deren Maßstäbe und Deutungen sie jedoch zumindest in bestimmten Bereichen ablehnen. Die Medienberichterstattung über spektakuläre Protestaktionen setzt nun nicht immer vorrangig deren inhaltliche Positionen in den Fokus, sondern maßgeblich ihre deviante Form. Diese Handlungen haben das Potenzial, als normverletzend bis hin zu kriminell dargestellt und entsprechend von der Gesamtgesellschaft als ablehnungswürdig und illegitim klassifiziert zu werden. Die Chance der Strategischen Kommunikation sozialer Bewegungen liegt nun in einem Reframing ihres Handelns und in der Inszenierung funktionaler Devianz. Hierdurch wird ihr innovatives Moment betont und herausgestellt, dass ihr Handeln einen Mehrwert für die Gesamtgesellschaft bietet. Dies begünstigt eine Solidarisierung mit dem »Underdog « und dessen Anliegen. Gelingt auch hier ein Wandel des Framings hin zu einer neuen geteilten Problembeschreibung, können so gesamtgesell- 
schaftliche Energien für wirkliche »Bewegung«freigesetzt und Erwartungen gemeinsam neu ausgehandelt werden.

\section{Literatur}

Ahlemeyer, Heinrich W. 1995. Soziale Bewegungen als Kommunikationssystem. Wiesbaden: VS Verlag für Sozialwissenschaften.

Altmeppen, Klaus-Dieter, Ulrike Röttger, und Günter Bentele. 2004. Public Relations und Journalismus: Eine lang andauernde und interessante »Beziehungskiste«. In: Schwierige Verhältnisse, herausgegeben von Klaus-Dieter Altmeppen, Ulrike Röttger und Günter Bentele, 7-15. Wiesbaden: VS Verlag für Sozialwissenschaften.

Baerns, Barbara. 1991. Öffentlichkeitsarbeit oder Journalismus: Zum Einfluß im Mediensystem. Köln: Wissenschaft und Politik.

Becker, Howard S. 2014. Außenseiter. Wiesbaden: Springer VS.

Bock, Christian. 2017. Ende_Gelände_2017_CHB_23. https://www.flickr.com/ photos/133937251@N05/26439720559/in/album-72157688093187921/.Zugegriffen: 25. März 2021

Bohnsack, Ralf. 2009. Qualitative Bild- und Videointerpretation: Die dokumentarische Methode. Opladen: Budrich.

Bonfadelli, Heinz, und Thomas N. Friemel. 2001. Medienwirkungsforschung. 2., überarbeitete Auflage. Konstanz: UVK.

Boyle, Michael P., Douglas M. McLeod, und Cory L. Armstrong. 2012. Adherence to the Protest Paradigm. The International Journal of Press/Politics 17 (2): 127144. DOI: https://doi.org/10.1177/1940161211433837.

Buchmeier, Yosuke. 2020. Towards a Conceptualization and Operationalization of Agenda-Cutting: A Research Agenda for a Neglected Media Phenomenon. Journalism Studies 21 (14): 2007-2024. DOI: https://doi.org/10.1080/1461670X. 2020.1809493.

Burri, Regula Valérie. 2008. Bilder als soziale Praxis: Grundlegungen einer Soziologie des Visuellen. Zeitschrift für Soziologie 37 (4): 342-358.

Colistra, Rita F. 2018. Power Pressures and Pocketbook Concerns: Perceptions of Organizational Influences on News Content in the Television Industry. International Journal of Communication 12: 1790-1810.

Crary, Jonathan. 2002. Aufmerksamkeit: Wahrnehmung und moderne Kultur. Frankfurt am Main: Suhrkamp.

Dalkowski, Sebastian. 2018. Der dauerbesetzte Wald. Rheinische Post vom 14. September, https://rp-online.de/nrw/panorama/hambacher-forst-chronologieder-ereignisse_aid-32968789. Zugegriffen: 25. März 2021.

Di Cicco, Damon T. 2010. The Public Nuisance Paradigm: Changes in Mass Media Coverage of Political Protest since the 1960s. Journalism \& Mass Communication Quarterly87(1):135-153.DOI:https://doi.org/10.1177/107769901008700108.

Ebert, Helmut, und Manfred Piwinger. 2007. Impression Management: Die Notwendigkeit der Selbstdarstellung. In: Handbuch Unternehmenskommunikation, 
herausgegeben von Manfred Piwinger und Ansgar Zerfaß, 205-225. Wiesbaden: Gabler.

Ehl, David, und Lisa Hänlein. 2019. Extinction Rebellion: Wie weit darf Protest gehen? Deutsche Welle vom 6. Oktober, https://p.dw.com/p/3PPnD. Zugegriffen: 25. März 2021.

Ertl, Sarah. 2015. Protest als Ereignis: Zur medialen Inszenierung von Bürgerpartizipation. Bielefeld: Transcript. DOI: http://dx.doi.org/10.14361/9783839430675.

Femers, Susanne. 2015. Public Relations aus sozialpsychologischer Sicht. In: Handbuch der Public Relations, herausgegeben von Romy Fröhlich, Peter Szyszka und Günter Bentele, 63-84. Wiesbaden: Springer VS.

Franck, Georg. 1998. Ökonomie der Aufmerksamkeit: Ein Entwurf. München: Hanser.

Freeman, R. Edward. 1984. Strategic Management: A Stakeholder Approach. Boston: Pitman Publishing.

Gamson, William A., und Gadi Wolfsfeld. 1993. Movements and Media as Interacting Systems. In: The Annals of the American Academy of Political and Social Science, herausgegeben von Richard D. Lambert und Alan W. Heston, 114-125. Newbury Park: Sage.

Gateau, Christophe. 2018. Räumung des Hambacher Forsts geht weiter - Aktivisten blockieren Kohlebahn. Spiegel Online vom 24. September, https://www.spiegel. $\mathrm{de}$ /panorama/hambacher-forst-raeumung-geht-weiter-aktivisten-blockierenkohlebahn-a-0604a3a1-a9ef-46cc-a2b8-0e1fcba5a5d9\#bild-5307098b-e9f44f74-83ac-647d33d82168. Zugegriffen: 25. März 2021.

Gerk, Andrea, und Henry Bernhard. 2019. Ist die Künstlergruppe ZPS eine kriminelle Vereinigung? Deutschlandfunk Kultur vom 3. April, https://www.deutschlandfunkkultur.de/zentrum-fuer-politische-schoenheit-ist-die-kuenstlergruppe.1013.de.html?dram:article_id=445408. Zugegriffen: 25. März 2021.

Goransson, Karina, und Anna-Sara Fagerholm. 2018. Towards visual strategic communications. JCOM 22 (1): 46-66. DOI: https://doi.org/10.1108/JCOM-122016-0098.

Görke, Alexander. 2008. Perspektiven einer Systemtheorie öffentlicher Kommunikation. In: Theorien der Kommunikations- und Medienwissenschaft, herausgegeben von Carsten Winter, Andreas Hepp und Friedrich Krotz, 173-191. Wiesbaden: VS Verlag für Sozialwissenschaften.

Hänggli, Regula. 2012. Key Factors in Frame Building. American Behavioral Scientist 56 (3): 300-317. DOI: https://doi.org/10.1177/0002764211426327.

Harlow, Summer. 2012. Social Media and Social Movements: Facebook and an Online Guatemalan Justice Movement That Moved Offline. New Media \& Society 14 (2): 225-243. DOI: https://doi.org/10.1177/1461444811410408.

Harlow, Summer, und Thomas J. Johnson. 2011. Overthrowing the Protest Paradigm? How The New York Times, Global Voices and Twitter Covered the Egyptian Revolution. International Journal of Communication 5: 1359-1374. https:// ijoc.org/index.php/ijoc/article/view/1239/611. Zugegriffen: 25. März 2021.

Herman, Edward S., und Noam Chomsky. 2002. Manufacturing Consent: The Political Economy of the Mass Media. New York: Pantheon. 
Hoffjann, Olaf. 2011. Vertrauen in Public Relations. Publizistik 56 (1): 65-84. DOI: https://doi.org/10.1007/s11616-010-0103-4.

Jacke, Christoph. 2004. Medien(sub)kultur: Geschichten - Diskurse - Entwürfe. Bielefeld: Transcript.

Jarren, Otfried, und Patrick Donges. 2011. Politische Kommunikation in der Mediengesellschaft. Wiesbaden: VS Verlag für Sozialwissenschaften.

Juris, Jeffrey S. 2012. Reflections on \#Occupy Everywhere: Social Media, Public Space, and Emerging Logics of Aggregation. American Ethnologist 39 (2): 259279. DOI: https://doi.org/10.1111/j.1548-1425.2012.01362.x.

Karmasin, Matthias, und Franzisca Weder. 2014. Stakeholder-Management als kommunikatives Beziehungsmanagement: Netzwerktheoretische Grundlagen der Unternehmenskommunikation. In: Handbuch Unternehmenskommunikation, herausgegeben von Ansgar Zerfaß und Manfred Piwinger, 81-103. Wiesbaden: Springer VS.

Kette, Sven, und Sebastian Barnutz. 2019. Was ist Compliance Management? Ein organisationstheoretischer Bestimmungs- und Einordnungsvorschlag. In: Compliance managen, herausgegeben von Sven Kette und Sebastian Barnutz, 7-22. Wiesbaden: Springer VS.

Kidd, Dustin, und Keith McIntosh. 2016. Social Media and Social Movements. Sociology Compass 10 (9): 785-794. DOI: https://doi.org/10.1111/soc4.12399.

Kim, Hyosun, und Tae Ho Lee. 2018. Strategic CSR Communication: A Moderating Role of Transparency in Trust Building. International Journal of Strategic Communication 12 (2): 107-124. DOI: https://doi.org/10.1080/1553118X.2018. 1425692.

Krüger, Uwe. 2016. Mainstream: Warum wir den Medien nicht mehr trauen. 2., durchgesehene und aktualisierte Auflage. München: C.H. Beck.

Krumpal, Ivar, und Roger Berger. 2020. Einleitung. In: Devianz und Subkulturen, herausgegeben von Ivar Krumpal und Roger Berger, 1-9. Wiesbaden: Springer VS.

Kühl, Stefan. 2011. Organisationen. Wiesbaden: VS Verlag für Sozialwissenschaften.

Lamnek, Siegfried. 1977. Kriminalitätstheorien, kritisch: Anomie u. Labeling im Vergleich. Kritische Information 60. München: Fink.

Lamnek, Siegfried. 2013. Theorien abweichenden Verhaltens I - »Klassische Ansätze«: Eine Einführung für Soziologen, Psychologen, Juristen, Journalisten und Sozialarbeiter. 9., durchgesehene Auflage. Paderborn: Fink.

Lauzen, Martha M. 1997. Understanding the Relation Between Public Relations and Issues Management. Journal of Public Relations Research 9 (1): 65-82. DOI: https://doi.org/10.1207/s1532754xjprr0901_03.

Luhmann, Niklas. 1991. Soziologie des Risikos. Berlin: de Gruyter.

Luhmann, Niklas. 1994. Systemtheorie und Protestbewegungen. Ein Interview. Forschungsjournal Neue Soziale Bewegungen 7 (2): 53-69.

Luhmann, Niklas. 1999. Funktionen und Folgen formaler Organisation. 5. Auflage. Berlin: Duncker \& Humblot.

Macnamara, Jim. 2014. A review of public communication by the mine owner and government departments and agencies during the 2014 Hazelwood Coal Mine 
Fire. University of Technology Sydney. http://hazelwoodinquiry.vic.gov.au/wpcontent/uploads/2014/08/Report-of-Prof-MacNamara.pdf. Zugegriffen: 25. März 2021.

Melenhorst, Lotte. 2015. The Media's Role in Lawmaking. The International Journal of Press/Politics 20 (3): 297-316. DOI: https://doi.org/10.1177/19401612155 81924.

Menzel, Birgit. 1997. Devianz im Wandel: Definitionstheoretisch orientierte Devianzsoziologie und die Gewaltthematik. Soziale Probleme 8 (2): 189-198.

Merten, Klaus (Hrsg.). 2009. Konstruktion von Kommunikation in der Mediengesellschaft. Wiesbaden: VS Verlag für Sozialwissenschaften.

Merten, Klaus, und Joachim Westerbarkey. 1994. Public Opinion und Public Relations. In: Die Wirklichkeit der Medien, herausgegeben von Klaus Merten, Siegfried J. Schmidt und Siegfried Weischenberg, 188-211. Wiesbaden: VS Verlag für Sozialwissenschaften.

Meyer, John W., und Brian Rowan. 1977. Institutionalized Organizations: Formal Structure as Myth and Ceremony. American Journal of Sociology 83 (2): 340363.

Möllers, Tobias. 2019. »Sie ist krank«: Merz kritisiert Gretas Auftritt vor der UN. Frankfurter Rundschau vom 28. September, https://www.fr.de/politik/friedrich-merz-kritisiert-gretas-auftritt-sie-sind-krank-13042471.html. Zugegriffen: 25. März 2021.

Neidhardt, Friedhelm, und Dieter Rucht. 1991. The Analysis of Social Movements: The State of the Art and Some Perspectives for Further Research. In: Research on Social Movements: The State of the Art in Western Europe and the USA, herausgegeben von Dieter Rucht, 421-464. Frankfurt am Main: Campus.

Oy, Gottfried. 1997. Gegenöffentlichkeit: Wandlungsprozesse linker Medienpolitik in der BRD. Forschungsjournal Neue Soziale Bewegungen 10 (3): 77-81.

Panofsky, Erwin. 1975. Ikonologie und Ikonographie. Eine Einführung in die Kunst der Renaissance. In: Sinn und Deutung in der bildenden Kunst, herausgegeben von Erwin Panofsky, 36-67. Köln: Dumont.

Peuckert, Rüdiger. 2016. Abweichendes Verhalten und soziale Kontrolle. In: Einführung in Hauptbegriffe der Soziologie, herausgegeben von Hermann Korte und Bernhard Schäfers, 127-151. Wiesbaden: Springer VS.

Philipps, Axel. 2012. Visual protest material as empirical data. Visual Communication 11 (1): 3-21. DOI: https://doi.org/10.1177/1470357211424675.

Popitz, Heinrich. 1961. Soziale Normen. European Journal of Sociology 2 (2): 185198. DOI: https://doi.org/10.1017/S0003975600000394.

Przyborski, Aglaja, und Thomas Slunecko. 2012. Linie und Erkennen: Die Linie als Instrument sozialwissenschaftlicher Bildinterpretation. Journal für Psychologie 20 (3): 1-37.

Rucht, Dieter. 1984. Zur Organisation der neuen sozialen Bewegungen. In: Politische Willensbildung und Interessenvermittlung: Verhandlungen der Fachtagung der DVPW vom 11.-13. Oktober 1983 in Mannheim, herausgegeben von Jürgen W. Falter, Christian Fenner und Michael T. Greven, 609-620. Wiesbaden: VS Verlag für Sozialwissenschaften. 
Rucht, Dieter. 1994. Modernisierung und neue soziale Bewegungen: Deutschland, Frankreich und USA im Vergleich. Frankfurt am Main: Campus.

Sandhu, Swaran. 2012. Public Relations und Legitimität: Der Beitrag des organisationalen Neo-Institutionalismus für die PR-Forschung. Wiesbaden: Springer VS.

Saxer, Ulrich. 2008. Gestaltung von Realität durch Public Relations. Communicatio Socialis 41 (4): 360-71. DOI: https://doi.org/10.5771/0010-3497-2008-4-360.

Schade, Henriette. 2018. Soziale Bewegungen in der Mediengesellschaft. Wiesbaden: Springer VS.

Schmitt-Beck, Rüdiger. 1998. Kommunikation (Neuer) Sozialer Bewegungen. In: Politische Kommunikation in der demokratischen Gesellschaft, herausgegeben von Otfried Jarren, Ulrich Sarcinelli und Ulrich Saxer, 473-481. Wiesbaden: VS Verlag für Sozialwissenschaften.

Scholl, Armin. 2009. Vom Dissens zur Dissidenz. Die Bedeutung alternativer Gegenöffentlichkeit für die Gesellschaft. In: Konstruktion von Kommunikation in der Mediengesellschaft, herausgegeben von Klaus Merten, 83-95. Wiesbaden: VS Verlag für Sozialwissenschaften.

Schulz, Winfried. 1979. Die Konstruktion von Realität in den Nachrichtenmedien: Analyse der aktuellen Berichterstattung. Freiburg: Alber.

Sewell, William H. 1992. A Theory of Structure: Duality, Agency, and Transformation. American Journal of Sociology 98 (1): 1-29.

Snow, David A., E. Burke Rochford, Steven K. Worden, und Robert D. Benford. 1986. Frame Alignment Processes, Micromobilization, and Movement Participation. American Sociological Review 51 (4): 464-481. DOI: https://doi. org/10.2307/2095581.

Stickler, Armin. 2005. NGOs und Soziale Bewegungen: Der Beitrag der Bewegungsforschung. In: Soziale Bewegungen und Global Governance: Eine Kritische Bestandsaufnahme, herausgegeben von Armin Stickler, 91-162. Bielefeld: Transcript.

Teune, Simon, und Moritz Sommer. 2017. Zwischen Emphase und Aversion: Großdemonstrationen in der Medienberichterstattung. https://protestinstitut.eu/ wp-content/uploads/2017/07/ipb-Forschungsbericht-Gro\%C3\%9Fdemonstrationen-in-der-Medienberichterstattung.pdf. Zugegriffen: 25. März 2021.

Valor, Carmen. 2005. Corporate Social Responsibility and Corporate Citizenship: Towards Corporate Accountability. Business \& Society Review 110 (2): 191-212. DOI: https://doi.org/10.1111/j.0045-3609.2005.00011.x.

Veneti, Anastasia. 2017. Aesthetics of Protest: An Examination of the Photojournalistic Approach to Protest Imagery. Visual Communication 16 (3): 279-298. DOI: https://doi.org/10.1177/1470357217701591.

Vliegenhart, Rens, Stefaan Walgra, Frank R. Baumgartner, Shaun Bevan, Christian Breuning, Sylvain Brourad, Laura Chaqués Bonafont. 2016. Do the media set the parliamentary agenda? A comparative study in seven countries. European Journal of Political Research 55 (2): 283-301. DOI: https://doi.org/10.1111/14756765.12134.

Walgrave, Stefaan, und Peter van Aelst. 2006. The Contingency of the Mass Media's Political Agenda Setting Power: Toward a Preliminary Theory. Journal of Com- 
munication 56 (1): 88-109. DOI: https://doi.org/10.1111/j.1460-2466.2006. 00005.x.

Werder, Kelly Page, Howard Nothhaft, Dejan Verčič, und Ansgar Zerfaß. 2018. Strategic Communication as an Emerging Interdisciplinary Paradigm. International Journal of Strategic Communication 12 (4): 333-351. DOI: https://doi. org/10.1080/1553118X.2018.1494181.

Wimmer, Jeffrey. 2007. (Gegen-)Öffentlichkeit in der Mediengesellschaft. Wiesbaden: VS Verlag für Sozialwissenschaften.

\section{Open Access}

Dieser Beitrag erscheint unter der Creative-Commons-Lizenz CC BY-ND 3.0 DE: https://creativecommons.org/licenses/by-nd/3.0/de/. 